\title{
PRIMER REGISTRO DE MORTALIDAD INCIDENTAL DE UTA STANSBURIANA EN UN PAROUE EÓLICO EN MÉXICO
}

\author{
FIRST RECORD OF INCIDENTAL MORTALITY OF UTA STANSBURIANA IN A WIND FARM IN MEXICO.
}

\author{
Alejandro Romero-de la Mora', Minerva A. Uribe-Rivera', Jorge H. Valdez-Villavicencio ${ }^{2}$, \\ VERÓNICA H. LÓPEZ-FRAUSTO ${ }^{1}$ Y ALDO A. GUEVARA-CARRIZALES ${ }^{1 \%}$ \\ ${ }^{1}$ Facultad de Ciencias, Universidad Autónoma de Baja California, Carretera Tijuana-Ensenada, 22830, Ensenada, Baja California, México. \\ ${ }^{2}$ Conservación de Fauna del Noroeste, A.C., 22785, Ensenada, Baja California, México. \\ *Correspondence: aldo.guevara@uabc.edu.mx
}

Abstract.- We report the finding of two dead specimens of Uta stansburiana in a wind farm located in the town of La Rumorosa, Baja California. The mortality was associated with run-over on access roads and drowning in the sewer systems. The specimens were found in the period from August 2015 to August 2016 during the carcass search activities of the wildlife mortality monitoring program. These findings are the first records of mortality of lizards in a wind farm in Mexico.

Keywords.- Baja California, wind energy, reptiles, lizards.

Resumen.- Se reporta el hallazgo de dos especímenes muertos de Uta stansburiana en un parque eólico localizado en el poblado de La Rumorosa, Baja California. La mortalidad se asoció al atropellamiento en caminos de acceso y al ahogamiento en los sistemas de alcantarillas. Los especímenes fueron encontrados en el periodo de agosto de 2015 a agosto de 2016 durante las actividades de búsqueda de cadáveres del programa de monitoreo de mortalidad de vida silvestre. Estos hallazgos constituyen los primeros registros de mortalidad de lacertilios en un parque eólico en México.

Palabras clave.- Baja California, energía eólica, reptiles, lagartijas.

La energía eólica se ha constituido como una de las fuentes de energía renovable de mayor demanda y desarrollo alrededor del mundo y que menos impactos ambientales genera en comparación con otras fuentes renovables (Evans et al., 2019). Sin embargo, se ha documentado que la construcción y operación de los parques eólicos causa ciertos efectos negativos sobre la fauna silvestre, principalmente en aves y murciélagos (Hötker, 2006; Arnett, 2008; Morrison, 2009).

En el caso de la herpetofauna, existe escasa información sobre los efectos negativos de los parques eólicos, no obstante, se han identificado impactos potenciales en la fauna terrestre que surgen en las etapas de construcción, operación y mantenimiento. En estas etapas puede ocurrir la muerte directa o impactos indirectos asociados con la modificación del hábitat, construcción de caminos, procesamiento y transporte de materiales (Lovich \& Ennen, 2013). La construcción de caminos provoca fragmentación del hábitat y crea barreras potenciales para el movimiento e intercambio genético. Otro tipo de impacto es el ruido que se produce en la etapa operativa en los naceles, la estructura que aloja todos los elementos mecánicos y eléctricos del aerogenerador, o cuando las palas en movimiento entran en contacto con el viento. Además, la turbulencia generada en las torres, produce sonidos infrasónicos no audibles para los humanos. Se ha documentado que todas estas fuentes de ruido pueden afectar el comportamiento de algunos animales como la ardilla de California (Spermophilus beecheyi) que se comunica por medio de vocalizaciones que pueden ser enmascaradas por el ruido ambiental (Rabin et al., 2006).

Adicionalmente, el paso de la electricidad a través de los cables genera campos electromagnéticos y la exposición crónica de la fauna a éstos puede afectar su sistema nervioso, inmune, cardiovascular o reproductivo. Además de los efectos fisiológicos, la exposición a los campos electromagnéticos puede alterar la orientación de ciertas especies, entre ellos los reptiles (Lovich \& Ennen, 2013).

Abbasi y Abbasi (2000) sugieren que las áreas ubicadas en dirección al viento dentro de los parques eólicos, pueden presentar alteraciones en el patrón del viento, precipitación, evaporación, incrementar la temperatura de los lagos y 

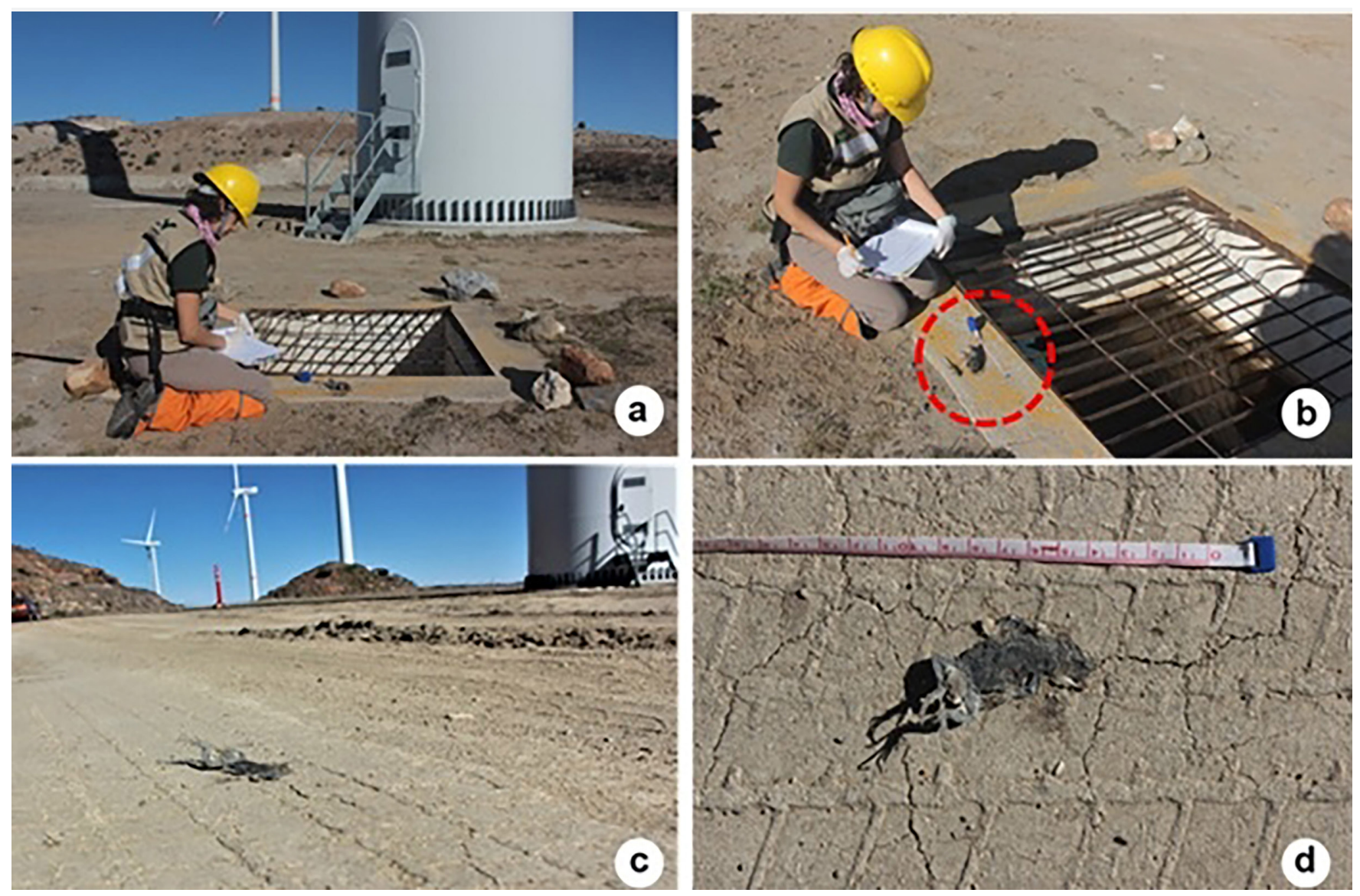

Figure 1. Specimens of Uta stansburiana found dead in the La Rumorosa Wind Farm: (a and b) drowned inside a sewer, (c and d) run-over on the main road.

Figura 1. Ejemplares de Uta stansburiana encontrados muertos en el Parque Eólico La Rumorosa: (a y b) ahogado dentro de una alcantarilla, (c y d) arrollado en el camino principal.

la humedad. Estos cambios pueden afectar a la fauna, especialmente a aquellas especies cuyo sexo se determina por la temperatura ambiental como en el caso de algunos reptiles. Por otra parte, la muerte de aves y murciélagos en parques eólicos, atrae a ciertos animales que consumen estos cadáveres, como los cuervos que son también depredadores de reptiles juveniles (Lovich \& Ennen, 2013).

El Parque Eólico La Rumorosa I (PER I) se ubica en el municipio de Tecate, Baja California $\left(32.49674^{\circ} \mathrm{N}, 116.08945^{\circ} \mathrm{O}\right.$, 1,365 $\mathrm{m}$ elev., datum WGS84). Este parque tiene una extensión de dos hectáreas y cinco aerogeneradores. El clima en la región es seco con lluvias de invierno y verano cálido (BSks) (CONABIO, 2015) con una temperatura media anual de $15.3^{\circ} \mathrm{C}$ y vegetación tipo chaparral de montaña (Delgadillo, 1998).

El monitoreo para determinar el efecto de la mortalidad de fauna silvestre se llevó a cabo durante el periodo de agosto de
2015 a agosto de 2016. Éste consistió en la búsqueda y registro de cadáveres en un polígono de $80 \mathrm{~m}^{2}$ trazado alrededor de cada uno de los cinco aerogeneradores y definido con base en la altura de las torres (California Energy Commission, 2007). Se recomienda definir así el área de búsqueda debido a que diversos estudios indican que más del $80 \%$ de los cadáveres de murciélagos son encontrados a una distancia que corresponde a la mitad de la altura máxima de la punta de la turbina al suelo (Kerns et al., 2005). Estos polígonos se recorrieron diariamente dos horas después del amanecer a través de 16 transectos a pie de 5 metros de ancho cada uno, con caminatas en patrón de zigzag de acuerdo con la metodología propuesta por Morrison et al. (2009). Los ejemplares colectados fueron medidos, identificados y depositados en la Colección Herpetológica de la Facultad de Ciencias de la Universidad Autónoma de Baja California ( $\mathrm{CH}-$ UABC) en Ensenada. 
Para la medición de los ejemplares se empleó un vernier digital graduado en milímetros y se tomaron las medidas estándares (Gregory, 2012) como la longitud hocico-cloaca (LHC), que se tomó de la punta de la nariz hasta la cloaca y longitud de la cola (LC), que se tomó de la base de la cloaca hasta la punta de la cola. Para la masa, se empleó una balanza de resorte marca Pesola ${ }^{\circledR}$ de $10 \mathrm{~g}$.

El 27 de febrero de 2016 se encontró el cadáver completo de un macho adulto de Uta stansburiana (CH-UABC 2227) ahogado dentro de una alcantarilla ubicada a $5 \mathrm{~m}$ al noreste de la base del quinto aerogenerador $\left(32.49313^{\circ} \mathrm{N}\right.$; $116.08825^{\circ} \mathrm{W}, 1370 \mathrm{~m}$ elevación; Fig. 1a y b). La alcantarilla estaba llena de agua hasta la mitad de su profundidad aproximadamente. Las medidas del ejemplar fueron: $\mathrm{LHC}=45.5 \mathrm{~mm} ; \mathrm{LC}=65 \mathrm{~mm}$, masa $=6.5 \mathrm{~g}$.

El 16 de marzo de 2016 se realizó el segundo hallazgo de otro espécimen adulto de U. stansburiana (CH-UABC 2228), arrollado en el camino de acceso principal al parque eólico, a $10 \mathrm{~m}$ al sur de la base del quinto aerogenerador $\left(32.49306^{\circ} \mathrm{N} ; 116.08808^{\circ} \mathrm{W}\right.$, 1,370 m elevación; Figura 1c y d). El espécimen no tenía cola y debido al mal estado del ejemplar no se pudo determinar el sexo ni determinar las medidas corporales o realizar la necropsia para determinar si el ejemplar murió por atropellamiento o fue arrollado después de haber muerto por otra causa.

Los registros aquí presentados constituyen los primeros reportes de mortalidad de reptiles en un parque eólico en México, y los primeros en lacertilios. Los únicos reportes de mortalidad de herpetofauna corresponden a ejemplares de la tortuga de desierto (Gopherus agassizzi) muertos por las mismas causas descritas en este trabajo (atropellamiento y entrampamiento en alcantarillas) (Lovich et al., 2011). Adicionalmente, la importancia de estos registros radica en que $U$. stansburiana es una especie categorizada como Amenazada en la NOM-SEMARNAT-059 (Diario Oficial de la Federación, 2010).

Con base en los registros de mortalidad de U. stansburiana aquí presentados, se implementó como medida de mitigación, el relleno de alcantarillas con grava y piedras para facilitar la salida de la fauna que pudiera quedar atrapada. A partir del uso de esta medida de manejo no se volvió a registrar la mortalidad por ahogamiento en los cinco meses restantes del monitoreo anual.

Acknowledgements.- Este trabajo se llevó a cabo con el financiamiento otorgado por Rufford Foundation y Fundación Jiji. Agradecemos al Cp. Francisco Javier Orduño director general de la Comisión Estatal de Energía del Estado de Baja California, Ing. Isaí Gómez y personal del Parque Eólico La Rumorosa I por las facilidades brindadas para la realización del presente trabajo. A dos revisores anónimos por su sugerencias para mejorar el manuscrito. A la SEMARNAT por el permiso de colecta GPA/ DGVS/0258.

\section{LITERATURE CITED}

Abbasi, S. A. \& N. Abbasi. 2000. The likely adverse environmental impacts of renewable energy sources. Applied Energy 65(1-4):21144.

Arnett, E., B. Erickson, W. Fiedler, J. Hamilton, H. Henry, A. Jain, G.D. Johnson, J. Kerns, R.R. Koford, C.P. Nicholson, T.J. O'connell, M.D. Piorkowski \& R.D. Tankersley Jr. 2008. Patterns of bat fatalities at wind energy facilities in North America. Journal of Wildlife Management 72:61-72.

California Energy Commission (CEC) and California Department of Fish and Game. 2007. California Guidelines for Reducing Impacts to Birds and Bats from Wind Energy Development. California Energy Commission, Renewables Committee, and Energy Facilities Siting Division, and California Department of Fish and Game, Resources Management and Policy Division, California.

CONABIO. 2019. Climas. Recuperado de http://www.conabio.gob. $\mathrm{mx} /$ informacion/gis/layouts/clima1mgw.png.

Delgadillo, J. 1998. Florística y ecología del norte de Baja California. México: Universidad Autónoma de Baja California. 407 p.

Diario Oficial de la Federación. 2010. Norma Oficial Mexicana NOM 059-ECOL-2010. Protección ambiental especies nativas de México de flora y fauna silvestres-Categorías de riesgo y especificaciones para su inclusión, exclusión o cambio - Lista de especies en riesgo. México.

Evans, A., V. Strezov \& T. Evans. 2009. Assessment of sustainability indicators for renewable energy technologies. Renewable and Sustainable Energy Reviews 13:1082-1088.

Gregory,P.T. 2012. Standard data from live specimens. Pp. 13814. En R.W. McDiarmid, M.S. Foster, C. Guyer, J.W. Gibbons, N. Chernoff (Eds.), Reptile biodiversity: standard methods for inventory and monitoring. University of California Press, Ltd., Berkeley, California, USA.

Hötker, H., K. Thomsen \& H. Jeromin. 2006. Impacts on biodiversity of exploitation of renewable energy resources: the example of 
birds and bats - facts, gaps in knowledge, demands for further research, and ornithological guidelines for the development of renewable energy exploitation. Michael-Otto-Institut im NABU. Bergenhusen, $65 \mathrm{p}$.

Kerns, J., W. P. Erickson \& E. B. Arnett. 2005. Bat and Bird Fatality at Wind Energy Facilities in Pennsylvania and West Virginia. Pp 2495. En E. B. Arnett (Ed.). Relationships between Bats and Wind Turbines in Pennsylvania and West Virginia: An Assessment of Bat Fatality Search Protocols, Patterns of Fatality, and Behavioral Interactions with Wind Turbines, final report submitted to the Bats and Wind Energy Cooperative, Bat Conservation International. Austin, Texas.

Lovich, J.E. \& J.R. Ennen. 2013. Assessing the state of knowledge of utility-scale wind energy development and operation on nonvolant terrestrial and marine wildlife. Applied Energy 103:52-60.
Lovich, J.E., J.R. Ennen, S. Madrak, B. Grover. 2011. Turtles and culverts, and alternative energy development: an unreported but potentially significant mortality threat to the Desert Tortoise (Gopherus agassizii). Chelonian Conservation and Biology 1:124129.

Morrison, M.L., K.C. Sinclair \&C.L. Thelander. 2009. Protocolo de muestreo para estudiar la influencia de los parques eólicos sobre aves y otros animales. Pp. 105-121. En M. Lucas, y M. Ferrer (Eds.). Aves y parques eólicos, valoración del riesgo y atenuantes. Madrid, España.

Rabin, L.A., R.G. Coss \& D.H. Owings. 2006. The effects of wind turbines on antipredator behavior in California ground squirrels (Spermophilus beecheyi). Biological Conservation 131:410-420. 